\title{
A new class of graceful graphs: $k$-enriched fan graphs and their characterisations
}

\author{
M. HAVIAR ${ }^{1,2}$ \\ S. KuRTULÍK ${ }^{1}$ \\ 1 Department of Mathematics, \\ Faculty of Natural Sciences, Matej \\ Bel University, Banská Bystrica, \\ Slovakia. \\ miroslav.haviar@umb.sk \\ kurtuliksamuel@gmail.com \\ ${ }^{2}$ Mathematical Institute, Slovak \\ Academy of Sciences, Bratislava, \\ Slovakia.
}

\begin{abstract}
The Graceful Tree Conjecture stated by Rosa in the mid 1960s says that every tree can be gracefully labelled. It is one of the best known open problems in Graph Theory. The conjecture has caused a great interest in the study of gracefulness of simple graphs and has led to many new contributions to the list of graceful graphs. However, it has to be acknowledged that not much is known about the structure of graceful graphs after 55 years.

Our paper adds an infinite family of classes of graceful graphs to the list of known simple graceful graphs. We introduce classes of $k$-enriched fan graphs $k F_{n}$ for all integers $k, n \geq 2$ and we prove that these graphs are graceful. Moreover, we provide characterizations of the $k$-enriched fan graphs $k F_{n}$ among all simple graphs via Sheppard's labelling sequences introduced in the 1970 s, as well as via labelling relations and graph chessboards. These last approaches are new tools for the study of graceful graphs introduced by Haviar and Ivaška in 2015. The labelling relations are closely related to Sheppard's labelling sequences while the graph chessboards provide a nice visualization of graceful labellings. We close our paper with an open problem concerning another infinite family of extended fan graphs.
\end{abstract}

\section{RESUMEN}

La Conjetura del Árbol Amable enunciada por Rosa a mediados de los 1960s dice que cada árbol puede ser etiquetado amablemente. Es uno de los problemas abiertos mejor conocidos en Teoría de Grafos. La conjetura ha causado un gran interés en el estudio de la amabilidad de grafos simples y ha llevado a muchas contribuciones nuevas a la lista de grafos amables. De todas formas, debe reconocerse que no se sabe mucho acerca de la estructura de grafos amables tras 55 años.

Nuestro artículo añade una familia infinita de clases de grafos amables a la lista de grafos amables simples conocidos. Introducimos clases de grafos abanico $k$-enriquecidos $k F_{n}$ para todos los enteros $k, n \geq 2$ y demostramos que estos grafos son amables. Más aún, entregamos caracterizaciones de los grafos abanico $k$-enriquecidos $k F_{n}$ entre todos los grafos simples vía sucesiones de etiquetado de Sheppard introducidas en los 1970s, y también a través de relaciones de etiquetados y tableros de ajedrez de grafos. Estos últimos acercamientos son herramientas nuevas para el estudio de grafos amables introducidos por Haviar e Ivaška en 2015. Las relaciones de etiquetado están relacionadas cercanamente con las sucesiones de etiquetado de Sheppard mientras que los tableros de ajedrez de grafos proveen una linda visualización de etiquetados amables. Concluimos nuestro artículo con un problema abierto relacionado con otra familia infinita de grafos abanico extendidos.

Keywords and Phrases: graph, graceful labelling, graph chessboard, labelling sequence, labelling relation.

2020 AMS Mathematics Subject Classification: 05C78

\section{(cc) BY-NC}




\section{Introduction}

A simple graph of size $m$ has a graceful labelling, and it is said to be graceful, when its vertices can be assigned different labels from the set $\{0,1, \ldots, m\}$ such that the absolute values of the differences in vertex labels of edges form the set $\{1, \ldots, m\}$. The Graceful Tree Conjecture stated by Rosa in [9] and [10] says that every tree is graceful. The conjecture has now been open for more than a half century and is one of the most attractive open problems in Graph Theory. The best source of information on attacks of the conjecture and on the study of labellings of graphs is the electronic book by Gallian [2].

Still, not much is known about the structure of graceful graphs. One of the known results, due to Hrnčiar and Haviar [6], is that all trees of diameter five are graceful.

The Graceful Tree Conjecture has led to a much increased interest in the study of gracefulness of simple graphs. In this paper we introduce a new infinite family of classes of simple graphs, $k$ enriched fan graphs $k F_{n}$, for all integers $k, n \geq 2$, and we show that they are graceful. These classes of graphs have been recently considered in the second author's MSc thesis [8]. The $k$-enriched fan graphs $k F_{n}$ are a natural generalisation of the class of fan graphs $F_{n}$, which were shown to be graceful in [5].

For a better understanding of the general $k$-enriched fan graphs and their characterisations, the special cases $k=2$ and $k=3$ of double and triple fan graphs are dealt with separately. Characterisations of the double and triple fan graphs, and then of the $k$-enriched fan graphs, are presented using the tools of labelling sequences, labelling relations and graph chessboards. Labelling sequences were introduced in 1976 by Sheppard in [11] while the labelling relations and graph chessboards as new tools for the study of graceful graphs were introduced and studied rather recently by Haviar and Ivaška in [5].

The basic terms and facts needed in this paper are presented in Section 2. This includes the concepts of labelling sequences, labelling relations and graph chessboards. In Section 3 we describe fan graphs and their graceful labellings from [5]. Then we generalise the concept of a fan graph to the one called a double fan graph resp. triple fan graph by connecting $n$ paths $P_{2}$ resp. $P_{3}$ to the main path of a given fan graph $F_{n}$. We construct for these graphs their graceful labellings and describe them by the corresponding labelling sequences, the labelling relations and the graph chessboards. Finally we study the general case of the $k$-enriched fan graphs $k F_{n}$ for any integers $k, n \geq 2$. These graphs contain, compared to the classic fan graphs $F_{n}, n$ copies of the star $S_{k}$ connected to the main path $P_{n}$. Again, we construct for these graphs their graceful labellings and characterize them by their labelling sequences, the labelling relations and the graph chessboards.

We conclude the paper with proposing another natural extension of the class of fan graphs and raising an open problem whether these extended fan graphs can be shown to be graceful and 
characterized in the manner presented here.

\section{Preliminaries}

In this section we recall the necessary basic terms concerning graph labellings as well as the concepts of labelling sequences, labelling relations and simple chessboards, which we use as our tools to describe new classes of graceful graphs. These definitions are taken primarily from [10] and [5].

In this paper we study only finite simple graphs, that is, finite unoriented graphs without loops and multiple edges. The following concept was called valuation by Rosa in his seminal paper [10].

Definition $2.1([10,5])$. A vertex labelling (or labelling for short) $f$ of a simple graph $G=(V, E)$ is a one-to-one mapping of its vertex set $V(G)$ into the set of non-negative integers assigning to the vertices so-called vertex labels.

By the label of an edge $u v$ in the labelling $f$ we mean the number $|f(u)-f(v)|$, where $f(u), f(v)$ are the vertex labels of $u, v$. In this text we will denote by $f\left(V_{G}\right)$ the set of all vertex labels and by $f\left(E_{G}\right)$ the set of all edge labels in the labelling $f$ of a graph $G$.

Several types of graph labellings are known, e.g. $\alpha, \beta, \sigma, \rho$, which have become very well-known since the publication of Rosa's paper [10] in 1967, and further $\gamma, \delta, p, q$ introduced by Rosa in his dissertation thesis [9] in 1965. In this text we study only $\beta$-labellings called graceful labellings.

Definition $2.2([10,5])$. A graceful labelling (or $\beta$-labelling) of a graph $G=(V, E)$ of size $m$ is a vertex labelling with the following properties:

(1) $f\left(V_{G}\right) \subseteq\{0,1, \ldots, m\}$, and

(2) $f\left(E_{G}\right)=\{1,2, \ldots, m\}$.

The term graceful was given to $\beta$-labellings in 1972 by Golomb [4] and its immediate use was enhanced by a popularization by Gardner [3]. Hence a graceful labelling of a graph of size $m$ has vertex labels among the numbers $0,1, \ldots, m$ such that the induced edge labels are different and cover all values $1,2, \ldots, m$. In Figure 1 we can see some graceful graphs.

Each graceful graph can be represented by a sequence of non-negative integers. This was shown by Sheppard in [11] where he introduced the concept of a labelling sequence as follows:

Definition $2.3([11,5])$. For a positive integer $m$, a labelling sequence is the sequence of nonnegative integers $\left(j_{1}, j_{2}, \ldots, j_{m}\right)$, denoted $\left(j_{i}\right)$, where

$$
0 \leq j_{i} \leq m-i \quad \text { for all } i \in\{1,2, \ldots, m\}
$$



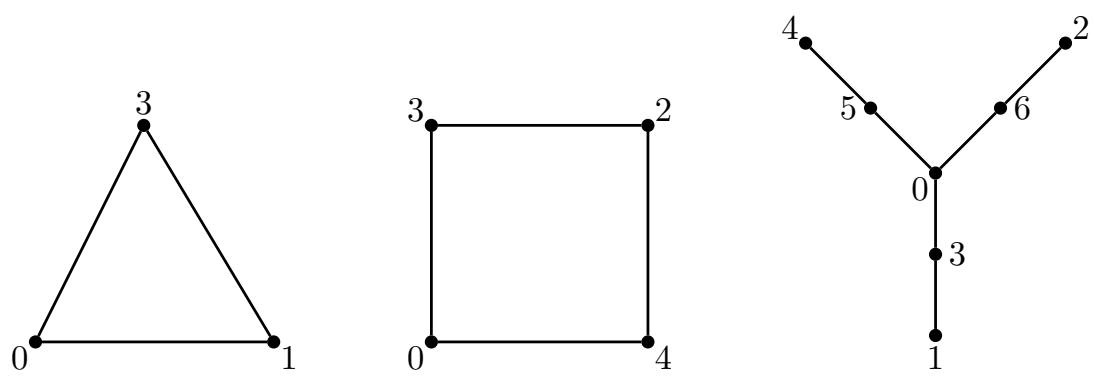

Figure 1: Some graceful graphs

Sheppard also proved that there is a one-to-one correspondence between graceful labellings of graphs (without isolated vertices) and labelling sequences. Therefore we can understand labelling sequences as a tool to encode graceful labellings of graphs. The connection is described in the following theorem.

Theorem $2.4([11,5])$. There exists a one-to-one correspondence between graphs of size $m$ having a graceful labelling $f$ and between labelling sequences $\left(j_{i}\right)$ of $m$ terms. The correspondence is given by

$$
j_{i}=\min \{f(u), f(v)\}, \quad i \in\{1,2, \ldots, m\},
$$

where $u, v$ are the end-vertices of the edge labelled $i$.

Now we recall the definition of a labelling relation. It is another tool to describe gracefully labelled graphs, which is closely related to the labelling sequence. The concept of the labelling relation was introduced and studied in [5].

Definition $2.5([5])$. Let $L=\left(j_{1}, j_{2}, \ldots, j_{m}\right)$ be a labelling sequence. Then the relation $A(L)=$ $\left\{\left[j_{i}, j_{i}+i\right], i \in\{1,2, \ldots, m\}\right\}$ is called a labelling relation assigned to the labelling sequence $L$.

To visualize a labelling relation and also a labelling sequence we will use a labelling table (see Figure 2). A table is formed by using the numbers $1,2, \ldots, m$ as headers for the columns, followed by two rows. The first row contains the numbers from the labelling sequence and the second row contains the sums of the corresponding numbers from the heading and from the first row. The pairs from first and second row in each column are then the elements of the labelling relation (and correspond to the edges of the graph).

\begin{tabular}{|c|c|c|c|c|}
\hline 1 & 2 & 3 & $\ldots$ & $m$ \\
\hline \hline$j_{1}$ & $j_{2}$ & $j_{3}$ & $\ldots$ & $j_{m}$ \\
\hline$j_{1}+1$ & $j_{2}+2$ & $j_{3}+3$ & $\ldots$ & $j_{m}+m$ \\
\hline
\end{tabular}

Figure 2: Displaying a labelling table

Example 2.6. In Figure 3 we can see the labelling table assigned to the labelling sequence $(5,4,3,2,1,0)$ and its graceful graph whose edges correspond to the elements of the labelling relation. 


\begin{tabular}{|l|l|l|l|l|l|}
\hline 1 & 2 & 3 & 4 & 5 & 6 \\
\hline \hline 5 & 4 & 3 & 2 & 1 & 0 \\
\hline 6 & 6 & 6 & 6 & 6 & 6 \\
\hline
\end{tabular}

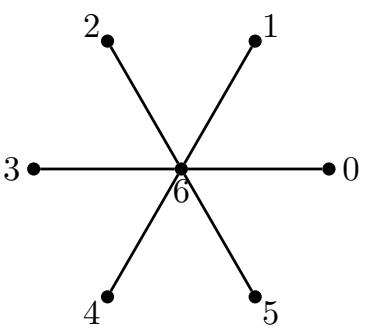

Figure 3: Example of a labelling table and its corresponding graceful graph

Every labelled simple graph of order $n$ can be represented by a chessboard, i.e. a table with $n$ rows and $n$ columns, where every edge labelled $u v$ is represented by a pair of dots with coordinates $[u, v]$ and $[v, u]$. This idea of visualization of vertex labellings of graphs by chessboards and independent discoveries of similar ideas are described in [5, Chapter 2]. One can also obtain such a graph chessboard by taking the adjacency matrix of a graph and placing dots in the cells corresponding to "ones" in the matrix while leaving the cells corresponding to "zeros" in the matrix empty (cf. [5, p. 25]).

Several types of graph chessboards like simple chessboards, double chessboards, M-chessboards, dual chessboards and twin chessboards were studied by Haviar and Ivaška in [5]. In this text we
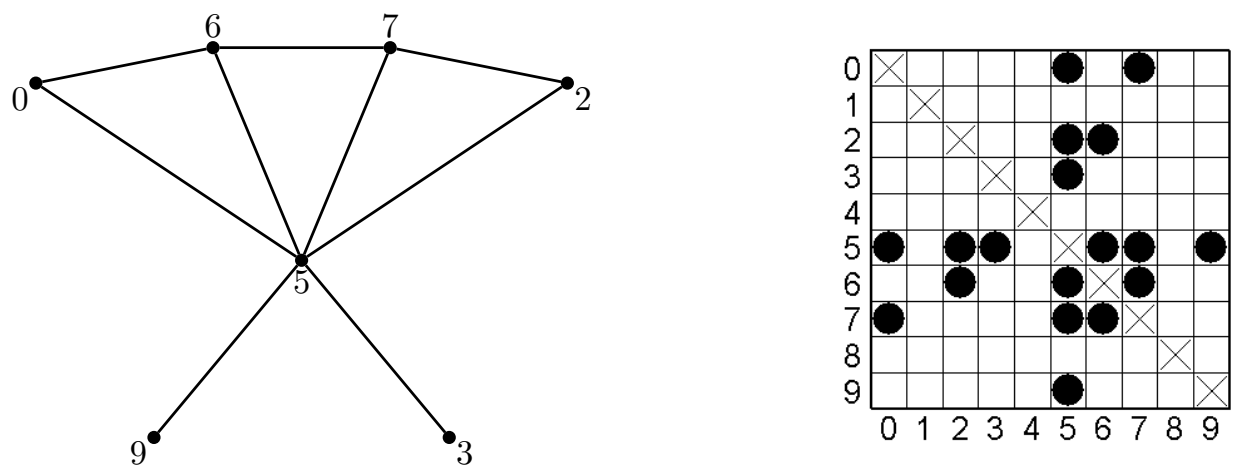

Figure 4: Example of a graph of size $m=9$ and its corresponding simple chessboard

use only the idea of a simple chessboard, which is a useful tool in the visualization of gracefully labelled graphs.

For a positive integer $m$ consider an $(m+1) \times(m+1)$-table. Rows are numbered by $0,1, \ldots, m$ from the top to the bottom and columns are numbered by $0,1, \ldots, m$ from the left to the right as shown in Figure 4. The cell with coordinates $[i, j]$ of the table will mean the cell in the $i$-th row and the $j$-th column. The $r$-th diagonal in the table is the set of all cells with coordinates $[i, j]$ where $i-j=r$ and $i \geq j$. The main diagonal is the 0 -th diagonal in the table and all other diagonals are called associate diagonals.

To a graph of size $m$ whose vertices are labelled by different numbers from the set $\{0,1,2, \ldots, m\}$ 
we assign its simple chessboard as the $(m+1) \times(m+1)$-table described above such that every edge labelled $u v$ in the graph is represented by a pair of dots in the cells with coordinates $[u, v]$ and $[v, u]$. It follows that the simple chessboards are symmetric about the main diagonal. An illustration of the simple chessboard of such a labelled graph of size 9 is in Figure 4.

If there is exactly one dot on each of the associate diagonals, then the simple chessboard will be called graceful as it clearly encodes a graceful graph. We can see a gracefully labelled graph of size 8 and its graceful simple chessboard in Figure 5.
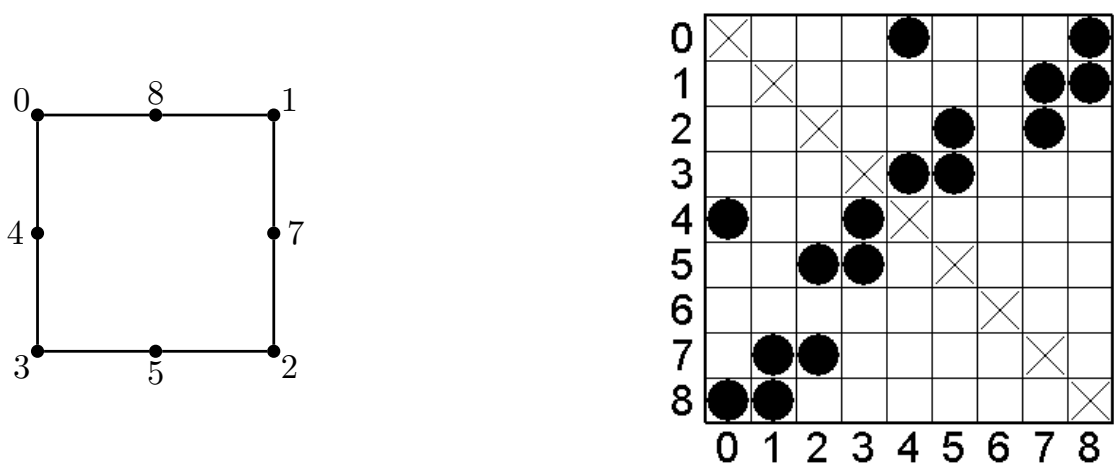

Figure 5: Gracefully labelled graph and its corresponding graceful simple chessboard

\section{$3 \quad$ Fan graphs and their descriptions}

A fan graph is a join of a path and a single vertex $K_{1}$. (See [5, Section 4.4.7] and Figure 6.) Its "bottom part" is formed by a star.

In this text we introduce the notation $F_{n}$ for a fan graph whose main path is $P_{n}$. We obviously require $n \geq 2$ in order to have the shape of "a fan". The fan graph $F_{n}$ thus has order $n+1$ and size $2 n-1$. In Figure 6 we can see a gracefully labelled fan graph $F_{8}$ of size 15 , with the main path $P_{8}$, its corresponding simple chessboard and its labelling relation.

The following two results taken from [5] characterize fan graphs via their labelling sequences, labelling relations and simple chessboards. (It is important to notice that [5, Section 4.4.7] considers the fan graphs that are $F_{n+1}$ in our present notation. In Figure 4.10 there we have the fan graph $F_{7}$ with considering $n=6$. That is why for our graphs $G$ studied in [5, Section 4.4.7] we talk about their size $m=2 n+1$.) The notation $\mathbb{N}$ in the next theorem and in our subsequent results is used, as in [5], for the set of all positive integers and the notation $\lfloor x\rfloor$ is used for the largest integer not greater than $x$ (the floor function).

Theorem $3.1([5])$. Let $G$ be a graph of size $m=2 n+1$ for some $n \in \mathbb{N}$. Then $G$ is a fan graph 

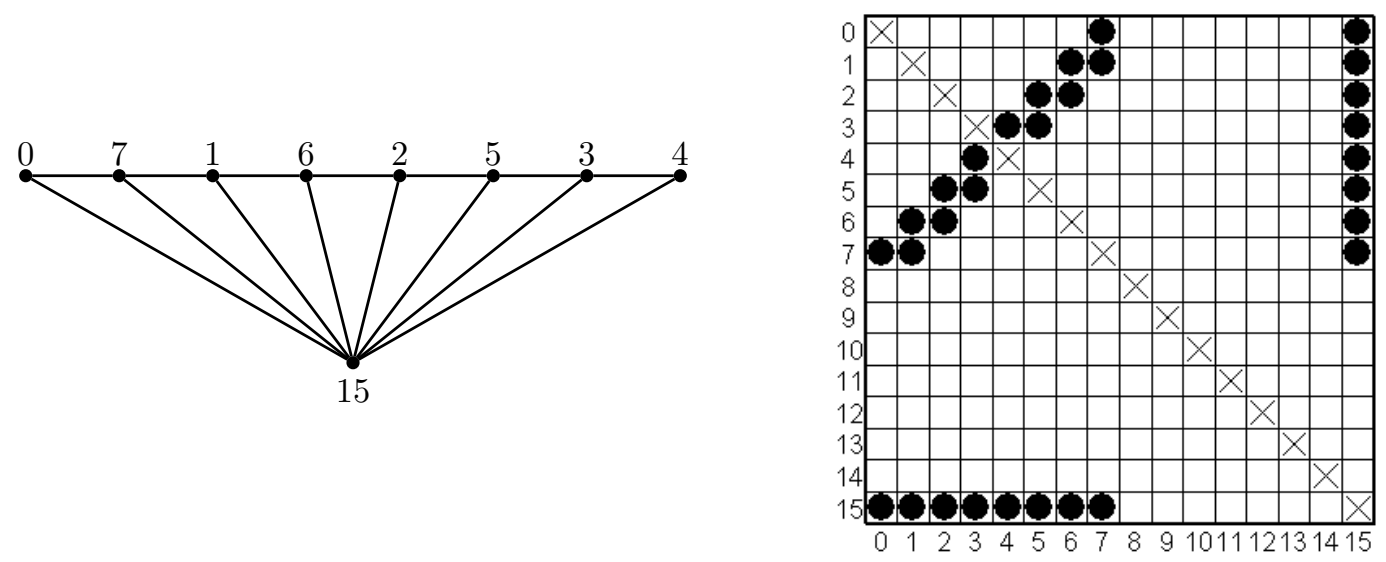

\begin{tabular}{|c|c|c|c|c|c|c|c|c|c|c|c|c|c|c|}
\hline 1 & 2 & 3 & 4 & 5 & 6 & 7 & 8 & 9 & 10 & 11 & 12 & 13 & 14 & 15 \\
\hline \hline 3 & 3 & 2 & 2 & 1 & 1 & 0 & 7 & 6 & 5 & 4 & 3 & 2 & 1 & 0 \\
\hline 4 & 5 & 5 & 6 & 6 & 7 & 7 & 15 & 15 & 15 & 15 & 15 & 15 & 15 & 15 \\
\hline
\end{tabular}

Figure 6: Representations of the gracefully labelled fan graph $F_{8}$

if and only if there exists a labelling sequence $\left(j_{1}, j_{2}, \ldots, j_{m}\right)$ of $G$ such that

$$
j_{i}= \begin{cases}\left\lfloor\frac{n-i+1}{2}\right\rfloor, & \text { if } i \leq n \\ m-i, & \text { if } i>n\end{cases}
$$

Corollary $3.2([5])$. Let $G$ be a graph of size $m=2 n+1$ that can be gracefully labelled and let $\mathcal{L}$ be the set of all its labelling sequences. Then the following are equivalent:

(a) $G$ is a fan graph.

(b) There exists a labelling sequence $L \in \mathcal{L}$ which satisfies (LSFG).

(c) For some $L \in \mathcal{L}$, the labelling relation is

$$
\begin{aligned}
A(L)= & \left\{[i, n-1] \mid i \in\left\{0,1, \ldots,\left\lfloor\frac{n-i}{2}\right\rfloor\right\}\right\} \cup \\
& \left\{[i, n-i+1] \mid i \in\left\{0,1, \ldots,\left\lfloor\frac{n}{2}\right\rfloor\right\}\right\} \cup \\
& \{[i, m] \mid i \in\{0,1, \ldots, n\}\} .
\end{aligned}
$$

(d) Some chessboard of $G$ looks like the chessboard in Figure 6.

Example 3.3. The sequence $(3,3,2,2,1,1,0,7,6,5,4,3,2,1,0)$ is the labelling sequence of the fan graph $F_{8}$ whose representations by the graph chessboard and the labelling table are seen in Figure 6. 


\section{Double and triple fan graphs and their descriptions}

By a double fan graph $D F_{n}$ we will mean the fan graph $F_{n}$ with extra $n$ pendant vertices, which are attached to the main path $P_{n}$. Hence double fan graphs $D F_{n}$ can be understood such that we connect $n$ paths $P_{2}$ to a given fan graph $F_{n}$ by identifying one vertex of each of the paths $P_{2}$ with one vertex of the main path $P_{n}$ of the fan graph. When we connect this way $n$ paths $P_{3}$ to a fan graph $F_{n}$, we get a triple fan graph $T F_{n}$.

In Figure 7 we see the double fan graph $D F_{5}$ on the left side and the triple fan graph $T F_{4}$ on the right side.
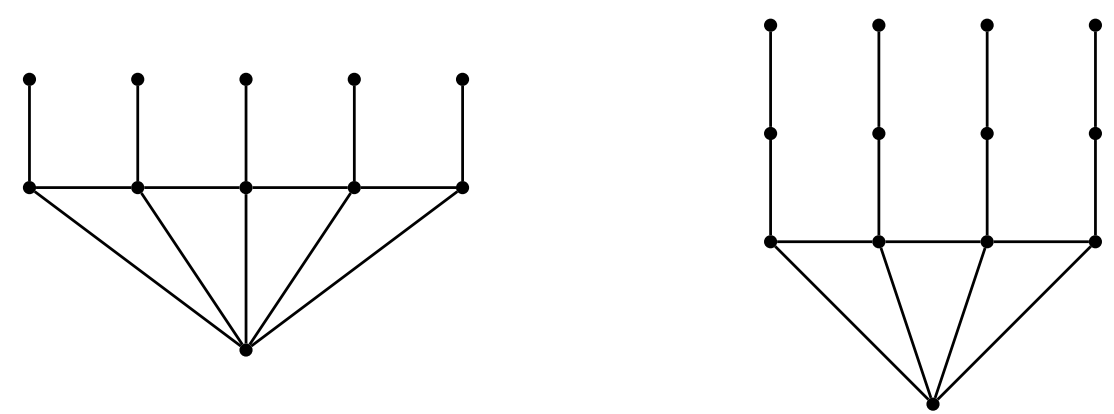

Figure 7: The double fan graph $D F_{5}$ (left) and the triple fan graph $T F_{4}$ (right)

We can divide vertices of such graphs into these groups: (i) the root vertex, which forms together with $n$ adjacent edges the "bottom" star, (ii) the "middle" vertices of the main path $P_{n}$, and (iii) the "upper" pendant vertices, which together with the "middle" vertices form $n$ paths $P_{2}$ resp. $P_{3}$ connected to the main path. Hence double fan graphs $D F_{n}$ resp. triple fan graphs $T F_{n}$ can be understood as certain extensions of the fan graphs by adding in the "upper part" $n$ paths $P_{2}$ (in double fan graphs) resp. $n$ paths $P_{3}$ (in triple fan graphs). We will often refer to the "bottom", "middle" and "upper parts" (in spite of the fact these parts are not pairwise disjoint and our naming of these parts refers only to our chosen visualization of these graphs) to assist in our proofs.

Example 4.1. The sequence $(2,1,1,0,0,1,2,3,4,4,3,2,1,0)$ is a labelling sequence of a gracefully labelled double fan graph $D F_{5}$ of size 14 and order 11 . The corresponding graph chessboard and labelling table are in Figure 8. Because the dots in the graph chessboard representing the edges of the mentioned "bottom", "middle" and "upper parts" of the double fan graph look respectively like the "bottom part", the "head" and the "neck" of a swan, we will refer to such chessboards as swan chessboards.

In the next theorems we characterise double fan graphs $D F_{n}$ and triple fan graphs $T F_{n}$ by their simple chessboards, labelling sequences and labelling relations. Within these characterisations we prove there are specific graceful labellings of these graphs, thus we show the graphs $D F_{n}$ and $T F_{n}$ 

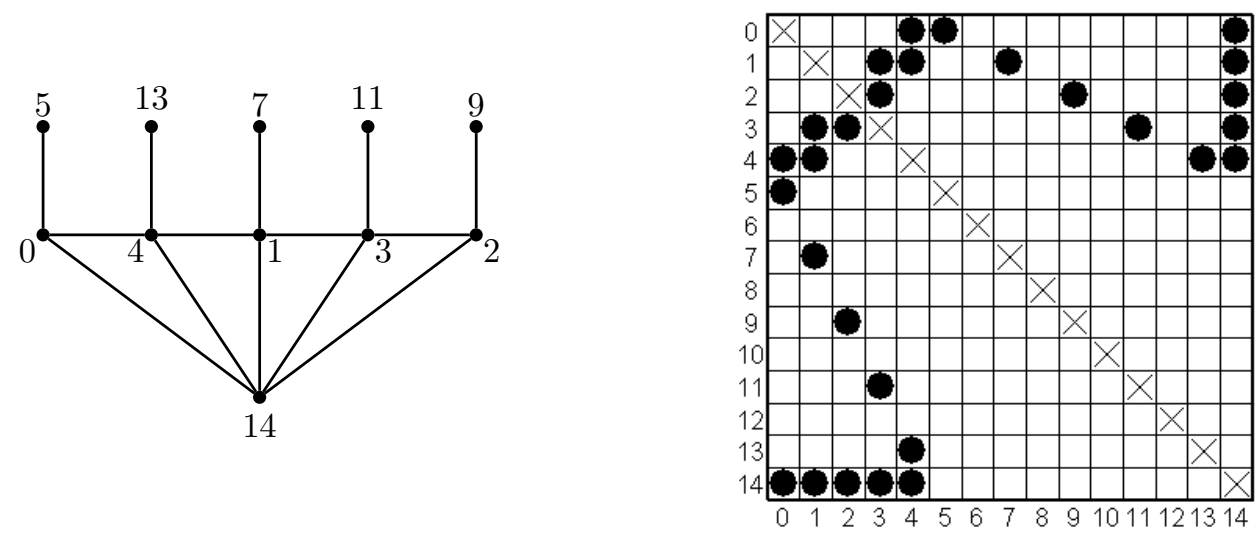

\begin{tabular}{|c|c|c|c|c|c|c|c|c|c|c|c|c|c|}
\hline 1 & 2 & 3 & 4 & 5 & 6 & 7 & 8 & 9 & 10 & 11 & 12 & 13 & 14 \\
\hline \hline 2 & 1 & 1 & 0 & 0 & 1 & 2 & 3 & 4 & 4 & 3 & 2 & 1 & 0 \\
\hline 3 & 3 & 4 & 4 & 5 & 7 & 9 & 11 & 13 & 14 & 14 & 14 & 14 & 14 \\
\hline
\end{tabular}

Figure 8: Representations of the gracefully labelled double fan graph $D F_{5}$

are graceful. So our double and triple fan graphs are new additions to the list of graceful graphs.

Theorem 4.2. Let $G$ be a graph of size $m=3 n-1$ for some $n \in \mathbb{N}-\{1\}$. Then the following are equivalent:

(1) $G$ is the double fan graph $D F_{n}$.

(2) There is a graceful labelling of $G$ with a swan chessboard.

(3) There exists a labelling sequence $L=\left(j_{1}, j_{2}, \ldots, j_{m}\right)$ of $G$ such that

$$
j_{i}= \begin{cases}\left\lfloor\frac{n-i}{2}\right\rfloor, & \text { if } i<n, \\ i-n, & \text { if } n \leq i<2 n \\ m-i, & \text { if } i \geq 2 n\end{cases}
$$

(4) There exists a labelling sequence $L$ of $G$ with the labelling relation

$$
\begin{aligned}
A(L)= & \{[u, v] \mid u, v \in\{0,1, \ldots, n-1\}, u<v, n-1 \leq u+v \leq n\} \cup \\
& \{[u, n+2 u] \mid u \in\{0,1, \ldots, n-1\}\} \cup \\
& \{[n-1-u, m] \mid u \in\{0,1, \ldots, n-1\}\} .
\end{aligned}
$$

Proof. (1) $\Rightarrow(2)$ Let $G$ be the double fan graph $D F_{n}$. We will label it in the following way. Let the labelling of the single vertex at the "bottom" (the root of $D F_{n}$ ) be $m$. Let the vertices of the middle path $P_{n}$ be labelled from one end-vertex of $P_{n}$ gradually by numbers $0, n-1,1, n-2,2, \ldots$ 
(see Figure 8). Hence the second end-vertex is labelled by $\left\lfloor\frac{n}{2}\right\rfloor$. Finally we will label $n$ pendant vertices in the "upper part". We start from the vertex which is attached to end-vertex, at which we started the labelling of the main path $P_{n}$. We label the vertices from this vertex gradually by numbers $n, m-1, n+2, m-3, n+4, \ldots$. We get edges with labellings $\{0, n\},\{n-1, m-1\},\{1, n+$ $2\},\{n-2, m-3\}$, and so on, in this "upper part". Now our labelling is done and we show this labelling is graceful with a corresponding swan chessboard (see Figure 8). One can easily verify the following statements:

(i) The "bottom part" of $G$ which is the star of size $n$ is in the chessboard represented by $n$ dots in the bottom row with coordinates $[m, 0],[m, 1], \ldots,[m, n-1]$ which form the "bottom of the swan".

(ii) The "middle part" of $G$ which is the path $P_{n}$ is in the chessboard represented by $n-1$ dots with coordinates $[n-1,0],[n-1,1],[n-2,1],[n-2,2], \ldots$ which form the "head of the swan".

(iii) The "upper part" of $G$ which is the union of $n$ paths $P_{2}$ is in the chessboard represented by $n$ dots with coordinates $[n, 0],[n+2,1],[n+4,2],[n+6,3], \ldots$ which form the "neck of the swan".

The described swan chessboard with three blocks of dots obviously has one dot on each diagonal. Hence $G$ is graceful.

$(2) \Rightarrow(3)$ Assume we have a graceful labelling of $G$ with a swan chessboard (as in Figure 8). We will verify that when we make the labelling sequence corresponding to this graceful labelling, we get exactly the labelling sequence satisfying (LSDFG). We use the distribution of dots in a swan chessboard into three blocks as in the previous part of the proof. So we consider the "head", the "neck" and the "bottom part" of a "swan". One can verify that these three blocks of dots in our chessboard can be assigned to the corresponding integers in the labelling sequence. The first block of dots, the "head", is represented in the corresponding labelling sequence by the integers $j_{i}$ of the form $\left\lfloor\frac{n-i}{2}\right\rfloor$ for $i<n$. The second block of dots, the "neck", is represented in the corresponding labelling sequence by the integers $j_{i}$ of the form $i-n$ for $n \leq i<2 n$. The third block of dots, the "bottom part", is represented in the corresponding labelling sequence by the integers $j_{i}$ of the form $m-i$ for $i \geq 2 n$.

We have shown that there exists a labelling sequence of $G$ satisfying the formula (LSDFG). $(3) \Rightarrow(4)$ Assume we have a labelling sequence $L$ of $G$ which satisfies (LSDFG). We will show that the labelling relation $A(L)$ from this labelling sequence consists of the pairs as described in (4). Indeed, one can verify that the non-negative integers $j_{i}$ from the labelling sequence, which have the form $\left\lfloor\frac{n-i}{2}\right\rfloor$ for $i<n$, correspond in $A(L)$ to the pairs $[u, v]$ where $u, v \in\{0,1, \ldots, n-1\}, u<v$ and $n-1 \leq u+v \leq n$. The next $j_{i}$ from the labelling sequence, which have the form $i-n$ for 
$n \leq i<2 n$, correspond to the pairs $[u, n+2 u]$ for $u \in\{0,1, \ldots, n-1\}$. Finally, $j_{i}$ from the labelling sequence, which have the form $m-i$ for $i \geq 2 n$, correspond to the pairs $[n-1-u, m]$ for $u \in\{0,1, \ldots, n-1\}$.

$(4) \Rightarrow(1)$ Assume that there exists a labelling sequence $L$ of $G$ with the labelling relation $A(L)$ as in (4). From the definition we know the labelled edges of $G$ correspond to the pairs in $A(L)$. One can verify that the pairs $[n-1-u, m]$ from $A(L)$ for $u \in\{0,1, \ldots, n-1\}$ correspond to the edges in "the bottom part" of graph $G$ which therefore is a star of size $n$. The pairs $[u, v]$ from $A(L)$ for $u, v \in\{0,1, \ldots, n-1\}, u<v$ and such that $n-1 \leq u+v \leq n$ correspond to the edges in "the middle part" of $G$ which therefore is the path $P_{n}$. Finally, the pairs $[u, n+2 u]$ for $u \in\{0,1, \ldots, n-1\}$ correspond to the edges in "the upper part" of $G$ which therefore form $n$ paths $P_{2}$ connected to the main path $P_{n}$. So the three parts in $A(L)$ correspond to the three parts of the double fan graph $D F_{n}$.

Hence a double fan graph can always be gracefully labelled so that its chessboard has the "head", the "neck" and the "bottom part" of a "swan". In the rest of this section we focus on a description of triple fan graphs.

Example 4.3. The sequence $(2,2,1,1,0,6,7,8,9,10,11,0,1,2,3,4,5,5,4,3,2,1,0)$ is a labelling sequence of a triple fan graph of size 23 and order 19. The corresponding graph diagram, graph chessboard and labelling table are in Figure 9.

Notice that while the swan chessboard of a double fan graph had one "neck" connecting the "head" and the "bottom part" of the "swan", the simple chessboard of a triple fan graph has 3 blocks of dots that look like a "swan without head", then "the head separated from the neck", and one extra "separated neck". Hence we will refer to such simple chessboards representing the triple fan graphs as 3-part swan chessboards.

The following characterisations of triple fan graphs can be proven using a method similar to that used in the previous theorem, and will be covered by the general case in the subsequent section. 

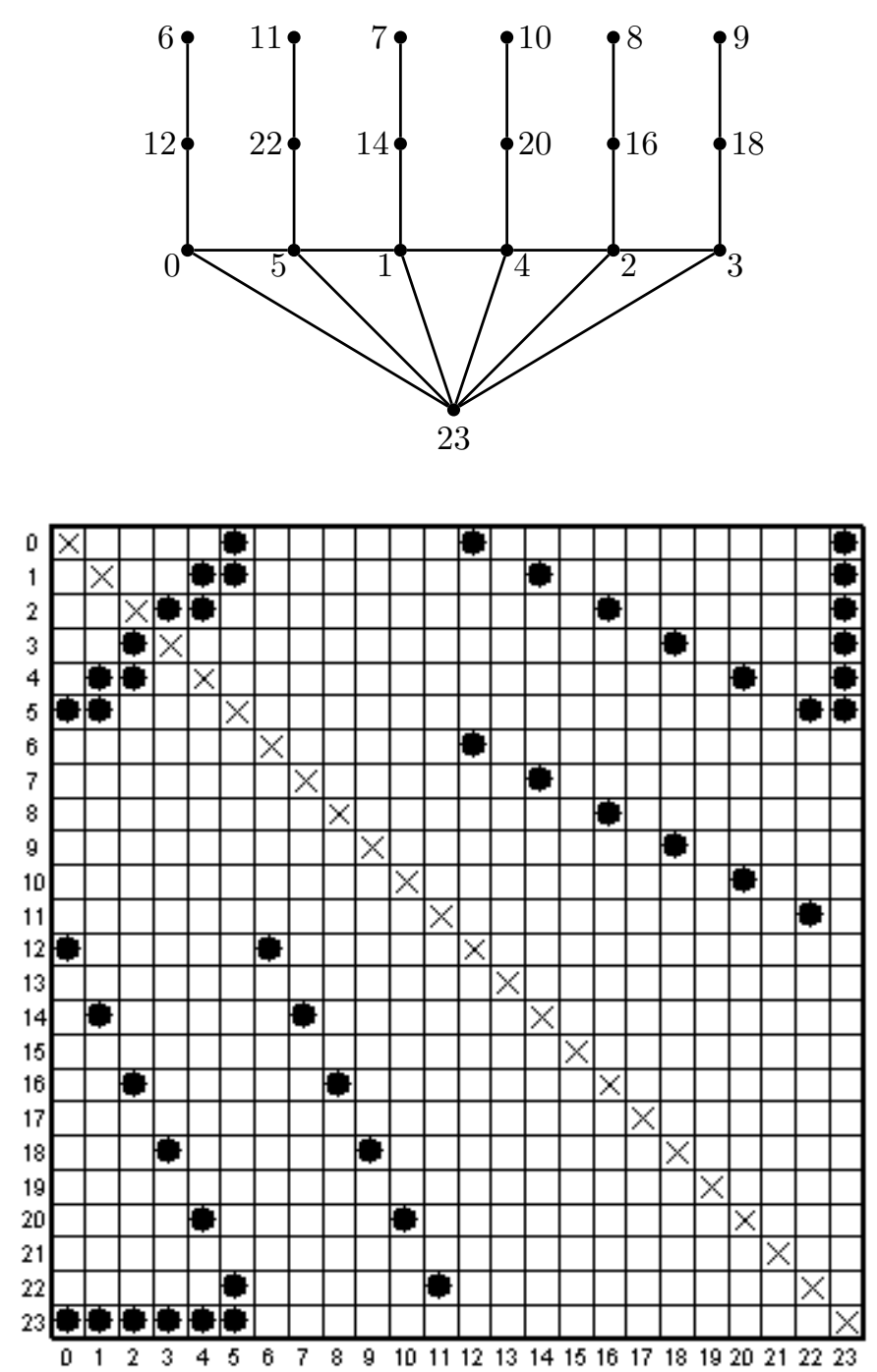

\begin{tabular}{|c|c|c|c|c|c|c|c|c|c|c|}
\hline 1 & 2 & 3 & 4 & 5 & 6 & 7 & 8 & 9 & 10 & 11 \\
\hline \hline 2 & 2 & 1 & 1 & 0 & 6 & 7 & 8 & 9 & 10 & 11 \\
\hline 3 & 4 & 4 & 5 & 5 & 12 & 14 & 16 & 18 & 20 & 22 \\
\hline
\end{tabular}

\begin{tabular}{|c|c|c|c|c|c|c|c|c|c|c|c|}
\hline 12 & 13 & 14 & 15 & 16 & 17 & 18 & 19 & 20 & 21 & 22 & 23 \\
\hline \hline 0 & 1 & 2 & 3 & 4 & 5 & 5 & 4 & 3 & 2 & 1 & 0 \\
\hline 12 & 14 & 16 & 18 & 20 & 22 & 23 & 23 & 23 & 23 & 23 & 23 \\
\hline
\end{tabular}

Figure 9: Representations of the gracefully labelled triple fan graph $T F_{6}$

Theorem 4.4. Let $G$ be a graph of size $m=4 n-1$ for some $n \in \mathbb{N}-\{1\}$. Then the following are equivalent:

(1) $G$ is the triple fan graph $T F_{n}$.

(2) There is a graceful labelling of $G$ with a 3-part swan chessboard. 
(3) There exists a labelling sequence $L=\left(j_{1}, j_{2}, \ldots, j_{m}\right)$ of $G$ such that

$$
j_{i}= \begin{cases}\left\lfloor\frac{n-i}{2}\right\rfloor, & \text { if } i<n, \\ i, & \text { if } n \leq i<2 n, \\ i-2 n, & \text { if } 2 n \leq i<3 n \\ m-i, & \text { if } i \geq 3 n .\end{cases}
$$

(4) There exists a labelling sequence $L$ of $G$ with the labelling relation

$$
\begin{aligned}
A(L)= & \{[u, v] \mid u, v \in\{0,1, \ldots, n-1\}, u<v, n-1 \leq u+v \leq n\} \cup \\
& \{[u, 2 u] \mid u \in\{n, n+1, \ldots, 2 n-1\}\} \cup \\
& \{[u, 2 n+2 u] \mid u \in\{0,1, \ldots, n-1\}\} \cup \\
& \{[n-1-u, m] \mid u \in\{0,1, \ldots, n-1\}\} .
\end{aligned}
$$

\section{General case: $k$-enriched fan graphs $k F_{n}$ and their descriptions}

According to the previous section we would assume that the more "separated necks" we have in the graph chessboard, the longer the paths in the "upper part" of the graph will be. Our work with Graph processor introduced in [7] and much used also in [5] has led us to a surprising observation that the "necks" in the graph chessboard do not represent in the corresponding graph the paths but the stars. We use for these graphs the term $k$-enriched fan graphs $k F_{n}$, where $k$ represents the order of the stars $S_{k}$ in the "upper part" of the graph and $n$ represents the order of the "middle" path $P_{n}$. Now we formally define this new term.

Definition 5.1. The $k$-enriched fan graph $k F_{n}$, for fixed integers $k, n \geq 2$, is the graph of size $(k+1) n-1$ obtained by connecting $n$ copies of the star $S_{k}$ of order $k$ to the fan graph $F_{n}$ such that one vertex of each copy of the star $S_{k}$ is identified with one vertex of the main path $P_{n}$ of $F_{n}$.

We notice that here the stars $S_{k}$ are connected to the main path $P_{n}$ of the fan graph $F_{n}$ exactly as in the previous section the paths $P_{2}$ (which are the stars $S_{2}$ ) resp. $P_{3}$ (the stars $S_{3}$ ) were connected to the main path $P_{n}$ of $F_{n}$ in the case of the double fan graphs $D F_{n}$ resp. triple fan graphs $T F_{n}$.

Example 5.2. In Figure 10 we see a gracefully labelled 4 -enriched fan graph $4 F_{6}$ obtained by connecting 6 copies of stars $S_{4}$ of order 4 to the fan graph $F_{6}$ as described above. The corresponding simple chessboard and labelling table are also in Figure 10. The labelling sequence of this graph is 
We notice that we can divide the vertices of the $k$-enriched fan graph $k F_{n}$ with any $k \geq 2$ similarly as in the case of double and triple fan graphs. Hence the graph consists of the "bottom part" (the root vertex and the adjacent edges), the "middle part" (the main path $P_{n}$ ) and the "upper part" (the disjoint union of $n$ stars $S_{k}$ ). The corresponding simple chessboard of the $k$-enriched fan graph $k F_{n}$ for $k \geq 3$ has $k$ blocks of dots that form a "swan without head", then "the separated head", and $k-2$ extra "separated necks". Hence we will refer to such simple chessboards representing the $k$-enriched fan graphs $k F_{n}$ as k-part swan chessboards.

Theorem 5.3. Let $G$ be a graph of size $m=(k+1) n-1$ for some fixed integers $k, n \geq 2$. Then the following are equivalent:

(1) $G$ is the $k$-enriched fan graph $k F_{n}$.

(2) There is a graceful labelling of $G$ with a $k$-part swan chessboard.

(3) There exists a labelling sequence $L=\left(j_{1}, j_{2}, \ldots, j_{m}\right)$ of $G$ such that

$$
j_{i}= \begin{cases}\left\lfloor\frac{n-i}{2}\right\rfloor, & \text { if } i<n, \\ i-n+(k-2) n, & \text { if } n \leq i<2 n, \\ i-n+(k-4) n, & \text { if } 2 n \leq i<3 n, \\ \vdots & \vdots \\ i-n+(k-2(k-1)) n, & \text { if }(k-1) n \leq i<k n, \\ m-i, & \text { if } i \geq k n .\end{cases}
$$

(4) There exists a labelling sequence $L$ of $G$ with the labelling relation $A(L)$ of the form

$$
\begin{aligned}
& \left\{\left[\left\lfloor\frac{n-i}{2}\right\rfloor,\left\lfloor\frac{n-i}{2}\right\rfloor+i\right] \mid i<n\right\} \cup \\
& \{[i-n+(k-2) n, 2 i-n+(k-2) n] \mid n \leq i<2 n\} \cup \\
& \{[i-n+(k-4) n, 2 i-n+(k-4) n] \mid 2 n \leq i<3 n\} \cup \\
& \vdots \\
& \{[i-n+(k-2(k-1)) n, 2 i-n+(k-2(k-1)) n] \mid(k-1) n \leq i<k n\} \cup \\
& \{[m-i, m] \mid i \geq k n\} .
\end{aligned}
$$

Proof. (1) $\Rightarrow(2)$ Let $G$ be the $k$-enriched fan graph $k F_{n}$. We will label the graph $G$ in a way similar to those labellings for the double and triple fan graphs. Let the labelling of the root of $k F_{n}$ be $m$. Let the vertices of the main path $P_{n}$ be labelled from one end-vertex of $P_{n}$ (we see it in Figure 10 from the left side) gradually by numbers $0, n-1,1, n-2, \ldots$ Hence the second end-vertex 

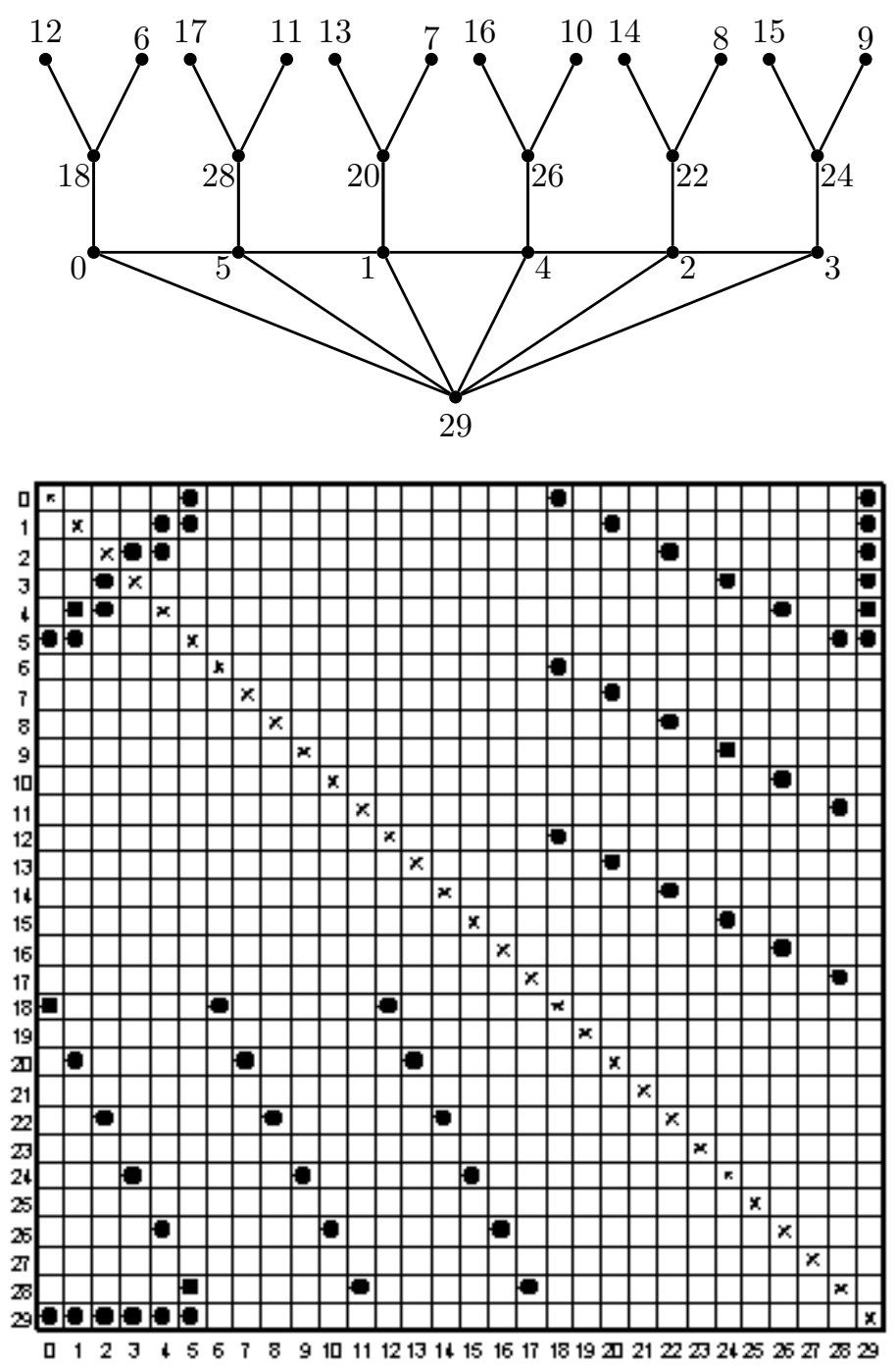

\begin{tabular}{|c|c|c|c|c|c|c|c|c|c|c|c|c|c|c|}
\hline 1 & 2 & 3 & 4 & 5 & 6 & 7 & 8 & 9 & 10 & 11 & 12 & 13 & 14 & 15 \\
\hline \hline 2 & 2 & 1 & 1 & 0 & 12 & 13 & 14 & 15 & 16 & 17 & 6 & 7 & 8 & 9 \\
\hline 3 & 4 & 4 & 5 & 5 & 18 & 20 & 22 & 24 & 26 & 28 & 18 & 20 & 22 & 24 \\
\hline
\end{tabular}

\begin{tabular}{|c|c|c|c|c|c|c|c|c|c|c|c|c|c|}
\hline 16 & 17 & 18 & 19 & 20 & 21 & 22 & 23 & 24 & 25 & 26 & 27 & 28 & 29 \\
\hline \hline 10 & 11 & 0 & 1 & 2 & 3 & 4 & 5 & 5 & 4 & 3 & 2 & 1 & 0 \\
\hline 26 & 28 & 18 & 20 & 22 & 24 & 26 & 28 & 29 & 29 & 29 & 29 & 29 & 29 \\
\hline
\end{tabular}

Figure 10: Representations of the gracefully labelled 4-enriched fan graph $4 F_{6}$

of $P_{n}$ is labelled by $\left\lfloor\frac{n}{2}\right\rfloor$. We start to label the central vertices of the $n$ stars $S_{k}$ in the "upper part" of the graph $G$ from the central vertex of $S_{k}$ attached to the vertex of the main path $P_{n}$ labelled by $n-1$. We label this vertex by $m-1$, the next central vertex of $S_{k}$ attached to the vertex $n-2$ will be labelled by $m-3$, the central vertex of $S_{k}$ attached to the vertex $n-3$ will be labelled by $m-5$ and so on, we end by labelling the central vertex of $S_{k}$ attached to the vertex 0 
by $m-2 n+1$ (see Figure 10). To label the remaining vertices of the $n$ stars $S_{k}$, we begin with the star, whose central vertex is labelled by $m-1$. The remaining vertices of this star are labelled by $((m-1)-(2 n-1)),((m-1)-(3 n-1)), \ldots$ It does not matter in what 'direction' we label these vertices. We proceed in the same way with labelling the remaining vertices of the other stars in the "upper part" of $G$, the role of the previous number $m-1$ is always played by the labelling of the central vertex of the given star (see Figure 10). Now our labelling of $G$ is done and we show that this labelling is graceful with a $k$-part swan chessboard.

For this we use a visualization via the corresponding simple chessboard of $G$ (see Figure 10). One can easily verify that:

(i) The "bottom part" of $G$ which is the star of size $n$ is in the simple chessboard represented by $n$ dots with coordinates $[m, 0],[m, 1], \ldots,[m, n-1]$ in the bottom row of the chessboard. (Exactly as for the double and triple fan graphs.)

(ii) The "middle part" of $G$ which is the main path $P_{n}$ is in the chessboard represented by $n-1$ dots with coordinates $[n-1,0],[n-1,1],[n-2,1],[n-2,2], \ldots$ which form a certain "separated head" in the case $k \geq 3$. (Exactly as for the triple fan graphs.)

(iii) The "upper part" of $G$ which is the union of $n$ stars $S_{k}$ is in the simple chessboard represented by $k-1$ "necks" each consisting of $n$ dots.

Hence the graph chessboard corresponding to our labelling is a $k$-part swan chessboard (as chessboard in Figure 10) and obviously it has exactly one dot on each diagonal. So the described labelling of $G$ is graceful.

$(2) \Rightarrow(3)$ Assume we have a graceful labelling of $G$ with a $k$-part swan chessboard. Consider the following $k$ blocks of dots of this chessboard: the "separated head", $k-1$ swan "necks" and the bottom row of the chessboard. One can verify that the "separated head" is represented in the corresponding labelling sequence by the integers $j_{i}$ having the form $\left\lfloor\frac{n-i}{2}\right\rfloor$ for $i<n$. The first "neck" from the right below the main diagonal is represented in the corresponding labelling sequence by the integers $j_{i}$ having the form $i-n+(k-2) n$ for $n \leq i<2 n$. The second "neck" from the right below the main diagonal is represented in the corresponding labelling sequence by the integers $j_{i}$ having the form $i-n+(k-4) n$ for $2 n \leq i<3 n$, and so on. Finally, the last "neck" (the first one from the left) is represented in the corresponding labelling sequence by the integers $j_{i}$ having the form $i-n+(k-2(k-1)) n$ for $(k-1) n \leq i<k n$. The bottom row of the chessboard is represented in the corresponding labelling sequence by the integers $j_{i}$ having the form $m-i$ for $i \geq k n$.

So we have shown that the labelling sequence corresponding to our $k$-part swan chessboard satisfies the formula (LSKFG). 
$(3) \Rightarrow(4)$ Assume there is a labelling sequence $L$ of $G$ which satisfies (LSKFG). One can verify that the corresponding labelling relation $A(L)$ consists of the pairs as described in (4). Indeed, the nonnegative integers $j_{i}$ from the labelling sequence having the form $\left\lfloor\frac{n-i}{2}\right\rfloor$ for $i<n$ correspond in $A(L)$ to the pairs $\left[\left\lfloor\frac{n-i}{2}\right\rfloor,\left\lfloor\frac{n-i}{2}\right\rfloor+i\right]$. The next integers $j_{i}$ from the labelling sequence, which have the form $i-n+(k-2) n$ for $n \leq i<2 n$, correspond in $A(L)$ to pairs $[i-n+(k-2) n, 2 i-n+(k-2) n]$. Further, the integers $j_{i}$ from the labelling sequence, which have the form $i-n+(k-4) n$ for $2 n \leq i<3 n$, correspond in $A(L)$ to pairs $[i-n+(k-4) n, 2 i-n+(k-4) n]$, and so on for the next parts of the labelling sequence, which correspond to the "necks". Finally, the integers $j_{i}$ from the labelling sequence, which have the form $m-i$ for $i \geq k n$, correspond to the pairs $[m-i, m]$.

$(4) \Rightarrow(1)$ Let $L$ be a labelling sequence of $G$ with the labelling relation $A(L)$ as in (4). The pairs $[m-i, m]$ in $A(L)$ for $i \geq k n$ correspond to the edges of the "bottom part" of $G$, which therefore is a star of order $n$. The pairs $\left[\left\lfloor\frac{n-i}{2}\right\rfloor,\left\lfloor\frac{n-i}{2}\right\rfloor+i\right]$ in $A(L)$ for $i<n$ correspond in the graph $G$ to the edges which form the "middle path" $P_{n}$. The remaining pairs in $A(L)$ correspond to the edges in "the upper part" of $G$. More precisely, each of the pairs $[i-n+(k-2) n, 2 i-n+(k-2) n]$ for $n \leq i<2 n$ corresponds to one edge in each of the $n$ "upper" stars $S_{k}$, each of the pairs $[i-n+(k-4) n, 2 i-n+(k-4) n]$ for $2 n \leq i<3 n$ corresponds to one of the (other) edges in each of the $n$ stars $S_{k}$ in the "upper part" of $G$, and so on, and finally, each of the pairs $[i-n+(k-2(k-1)) n, 2 i-n+(k-2(k-1)) n]$ for $(k-1) n \leq i<k n$ corresponds to the remaining edges in each of the $n$ stars $S_{k}$ in the "upper part" of $G$. So we get $n$ stars $S_{k}$ in the "upper part" of $G$. Thus we have in $G$ the "bottom" star of size $n$, the "middle" path $P_{n}$ and the $n$ "upper" stars $S_{k}$ connected to the vertices of the "middle" path $P_{n}$. Hence the graph $G$ is the $k$-enriched fan graph $k F_{n}$.

\section{Conclusion}

Studies of other extended fan graphs can be found in the literature. Some of them are seen in Figure 11 below. The first two graphs are taken from [1]. The author named the first one as a double fan graph, but it is different from our double fan graph as defined in this paper. It consists of two fan graphs that have a common main path. The second graph was obtained by adding some edges to a vertex of the main path.

One of the possibilities for other extensions of fan graphs would be adding paths of the same length $P_{k}$ (not stars $S_{k}$ as in our case) to the main path $P_{n}$ of the fan graph $F_{n}$. These graphs and our $k$-enriched fan graphs $k F_{n}$ would be the same for the cases $k=2$ and $k=3$. Such extended fan graphs, let us denote them as $P_{k} F_{n}$ (our $k$-enriched fan graphs $k F_{n}$ in this more universal notation would be denoted $S_{k} F_{n}$ ) can look like the third graph in Figure 11. This graph would be the extended fan graph $P_{4} F_{3}$ as the paths $P_{4}$ are connected to the main path of the fan graph $F_{3}$. We 
conclude our paper with the following open problem:

Problem 6.1. Are the extended fan graphs $P_{k} F_{n}$ (obtained by connecting in the described way $n$ paths $P_{k}$ to the main path $P_{n}$ of the fan graph $F_{n}$ ) graceful? And if so, is there a characterization of them via a certain "canonical" graph chessboard and the corresponding labelling sequence and the labelling relation like the characterizations of the graceful graphs presented in this paper?
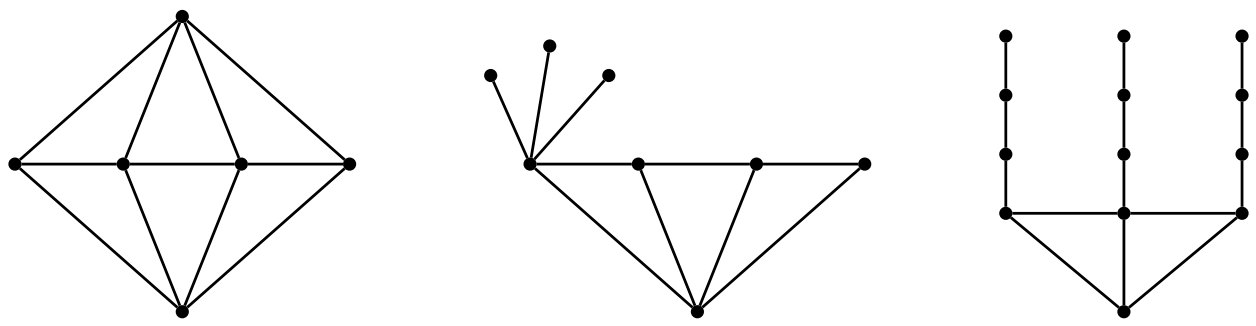

Figure 11: Other extended fan graphs

\section{Acknowledgements}

The first author acknowledges a support by Slovak VEGA grant 2/0078/20 and a Visiting Professorship at University of Johannesburg. Both authors acknowledge assisting remarks by Dr. Andrew P.K. Craig from the University of Johannesburg. The authors also thank the anonymous referee for pointing to a recent paper [12] where (generalized) comb-like trees are introduced which can be considered as new candidates for families of graceful graphs and then possibly also characterized in the manner presented in this paper. 


\section{References}

[1] S. Amutha and M. Uma Devi, "Super Graceful Labeling for Some Families of Fan Graphs", Journal of Computer and Mathematical Sciences, vol. 10, no. 8, pp. 1551-1562, 2019.

[2] J. A. Gallian, "A Dynamic Survey of Graph Labeling", Electron. J. Combin., \# DS6, Twentysecond edition, 2019.

[3] M. Gardner, "Mathematical Games: The graceful graphs of Solomon Golomb", Sci. Am., vol. 226, no. 23, pp. 108-112, 1972.

[4] S. W. Golomb, "How to number a graph", In: Graph Theory and Computing, pp. 23-37, Academic Press, 1972.

[5] M. Haviar and M. Ivaška, Vertex Labellings of Simple Graphs. Research and Exposition in Mathematics, vol. 34, Lemgo, Germany: Heldermann-Verlag, 2015.

[6] P. Hrnčiar and A. Haviar, "All trees of diameter five are graceful", Discrete Math., vol. 233, no. 1-3, pp. 133-150, 2001.

[7] M. Ivaška, "Chessboard Representations and Computer Programs for Graceful Labelings of Trees", Student Competition ŠVOČ, M. Bel University, Banská Bystrica, 2009.

[8] S. Kurtulík, "Graceful labellings of graphs", MSc. thesis, 52 pp., Department of Mathematics, M. Bel University, Banská Bystrica, 2020.

[9] A. Rosa, "O cyklických rozkladoch kompletného grafu" [On cyclic decompositions of the complete graph], PhD thesis (in Slovak), Československá akadémia vied, Bratislava, 1965.

[10] A. Rosa, "On certain valuations of the vertices of a graph", In: Theory of Graphs (Internat. Symposium, Rome, July 1966), pp. 349-355, New York: Gordon and Breach, 1967.

[11] D. A. Sheppard, "The factorial representation of major balanced labelled graphs", Discrete Math., vol. 15, no. 4, pp. 379-388, 1976.

[12] K. Xu, X. Li and S. Klavžar, "On graphs with largest possible game domination number", Discrete Math., vol. 341, no. 6, pp. 1768-1777, 2018. 